\title{
Implicaciones de la última reforma legislativa en materia de protección social del trabajo a tiempo parcial: Condiciones de acceso y efectos de su aplicación
}

\author{
Pilar Callau Dalmau \\ Departamento de Derecho de la Empresa. \\ Facultad de Ciencias Sociales y del Trabajo. \\ UNIVERSIDAD DE ZARAGOZA \\ pcallau@unizar.es
}

Resumen: Los cambios introducidos por el artículo 5 del Real Decreto-ley 11/2013, de 2 de agosto, para la protección de los trabajadores a tiempo parcial y otras medidas urgentes en el orden económico y social, como consecuencia de la Sentencia del Tribunal Constitucional 61/2013, de 14 de marzo, se concretan en un conjunto de medidas específicas, relativas a su acción protectora.

En este artículo se analizan las implicaciones directas que los mismos representan respecto al cómputo de los períodos de cotización necesarios para causar derecho a diferentes prestaciones, así 
como las consecuencias de las últimas actuaciones reformistas, en cuanto a la determinación de la cuantía de las pensiones de jubilación e incapacidad permanente derivada de enfermedad común, comprobando a partir de la simulación de casos concretos que originan datos adicionales a los conceptos claves examinados, que se hace necesario un replanteamiento de la posición del trabajador a tiempo parcial en el ámbito del sistema de la Seguridad Social, que no coincide exactamente con el planteado por el legislador en su última reforma, aún cuando formalmente en la misma, se asimila la protección social del trabajador a tiempo parcial con la del trabajador a tiempo completo.

Palabras clave: Trabajador a tiempo parcial, reforma, protección social, jubilación, incapacidad permanente, coeficiente de parcialidad, principio de asimilación.

\title{
Implications of the Latest Legislative Reform of Social Protection with respect to Part-time Work: Access Conditions and Effects of their Application.
}

\begin{abstract}
The amendments introduced by Article 5 of Executive Order 11/2013, of 2 August, for the protection of part-time workers and other urgent measures taken in the economic and social order as a consequence of the Ruling of the Constitutional Court 61/2013, of 14 March, are embodied in a set of specific measures relating to its protective action.

In this paper the direct implications are analysed with respect to the computation of the number of payments required to be entitled to various benefits, as well as the consequences of the latest legislative reforms, with respect to determining the amount of retirement pensions and permanent disability deriving from common illness, with verification being carried out by simulating specific cases that furnish further data to the key concepts examined. This makes it necessary to rethink the position of the part-time worker's position within the context of the Social Security system, which does not coincide exactly with the one contemplated by the legislator in the
\end{abstract}


latest reform, even when the social protection offered to the parttime worker is assimilated with that of the full-time worker.

Keywords: Part-time worker, reform, social protection, retirement, permanent disability. 



\section{Implicaciones de la última}

reforma legislativa en materia

de protección social del

trabajo a tiempo parcial:

Pilar

Callau Dalmau

\section{Condiciones de acceso y efectos de su aplicación.}

Recibido: 14/03/2017

Aceptado: 17/06/2017

\section{INTRODUCCIÓN}

El acceso a las prestaciones de Seguridad Social de los trabajadores a tiempo parcial (en adelante, TTP), así como la determinación de su cuantía, se encuentran regulados en los artículos 245 a 248 del Real Decreto Legislativo (en adelante, RDL) 8/2015, de 30 de octubre, por el que se aprueba el texto refundido de la Ley General de la Seguridad Social (en adelante, LGSS).

Estas disposiciones normativas, que fueron objeto de modificación por el artículo 5 del Real Decreto-ley (en adelante, RD-L) 11/2013, de 2 de agosto, para la protección de los TTP y otras medidas urgentes en el orden económico y social, introdujeron cambios importantes en este asunto'.

Las nuevas reglas relativas a la acción protectora de la Seguridad Social de este tipo de trabajadores ${ }^{2}$, fueron adoptadas tras la

${ }^{1}$ Que se recogen en los mismos términos, en el art.5 de la Ley 1/2014, de 28 de febrero, para la protección de los trabajadores a tiempo parcial y otras medidas urgentes en el orden económico y social.

${ }^{2}$ Concretamente, los trabajadores con contrato a tiempo parcial, contrato de relevo a tiempo parcial y contrato de trabajo fijo-discontinuo, de conformidad con 
Sentencia del Tribunal Constitucional (en adelante, STC) 61/2013, de 14 de marzo $^{3}$, al ser declarada su regulación inconstitucional y nula. Se entendía que vulneraba el artículo 14 CE por lesionar el derecho a la igualdad ${ }^{4}$, y también, a la vista de su predominante incidencia sobre el empleo femenino, por provocar una discriminación indirecta por razón de sexo ${ }^{5}$. En este sentido, el TC ha diferenciado jurídicamente varias vertientes de los principios de igualdad y no discriminación, realizando una interpretación conjunta de ambos, y distinguiendo, la aplicación del principio de igualdad ante la ley, el principio de igualdad en la aplicación de la ley y el derecho a no ser discriminado por motivos sociales y personales, mediante una discriminación directa o indirecta, que deriva en una condena sobre aquellas acciones que impidan hacer efectivo el derecho originario a la igualdad, permitiendo el establecimiento de acciones positivas en ciertos casos y bajo determinadas circunstancias (Nogueira, 2012: 21).

En este punto, es interesante recordar que en otras sentencias posteriores a la STC 61/2013, también se cuestiona el cálculo de los períodos de cotización en el caso de TTP, para acceder a las correspondientes prestaciones económicas, respecto de los periodos acreditados, incluidos los contratos de trabajo

lo establecido en los arts. 12 y 16 del RDL 2/2015, de 23 de octubre, por el que se aprueba el texto refundido de la Ley del Estatuto de los Trabajadores, comprendidos en el campo de aplicación del Régimen General, incluidos los trabajadores a tiempo parcial o fijos discontinuos pertenecientes al Sistema Especial para Empleados de Hogar, tal como se establece en el art. 245.2 LGSS.

${ }^{3}$ Resolviendo la cuestión de inconstitucionalidad planteada por la Sala de lo Social del Tribunal Superior de Justicia de Galicia núm. 5862-2003, de 11 de noviembre de 2003 , derivada del caso de una trabajadora a tiempo parcial, a la que le fue denegada una pensión contributiva de jubilación por no haber reunido el periodo mínimo de cotización de 15 años exigido para ello.

${ }^{4}$ Ya que el principio de igualdad no sólo exige que la diferencia de trato resulte objetivamente justificada, sino también que supere un juicio de proporcionalidad en sede constitucional sobre la relación existente entre la medida adoptada, el resultado producido y la finalidad pretendida, aludiendo en este sentido a las SSTC 63/2011, de 16 de mayo (FJ 3); 117/2011, de 4 de julio (FJ 4) y 79/2011, de 6 de junio (FJ 3).

${ }^{5}$ Según la define la doctrina del TC y la normativa comunitaria (art. 2.2 de la Directiva 97/80/CE, de 15 de diciembre de 1997, y art. 2.2 de la Directiva 2002/73/ CE, de 23 de septiembre de 2002), apoyada en una consolidada doctrina del Tribunal de Justicia de la Unión Europea, a lo que cabe añadir la prohibición de discriminación por razón del trabajo a tiempo parcial que inspira la regulación de la Directiva 97/81/CE, de 15 de diciembre de 1997. 
fijo-discontinuo a tiempo parcial o completo, con independencia de que la reducción de jornada se realice en cómputo diario, semanal, mensual o anual ${ }^{6}$.

Como consecuencia del fallo de la STC 61/2013, y cubriendo el vacío regulador producido, en el RD-L 11/2013, se recogen determinadas modificaciones que se introducen en la LGSS, en cuanto a la protección social del trabajo a tiempo parcial, y que se concretan en un conjunto de reglas específicas relativas a la acción protectora de la Seguridad Social aplicables a TTP, referentes a las contingencias de jubilación, incapacidad permanente (en adelante, IP), muerte y supervivencia, incapacidad temporal (en adelante, IT), maternidad y paternidad ${ }^{7}$, dejando al margen las reglas previstas para la prestación de desempleo ${ }^{8}$.

De esta manera, además de acomodarse a las exigencias constitucionales de igualdad y de no discriminación, en los términos previstos en el ordenamiento comunitario ${ }^{9}$, estas

${ }^{6}$ STC71/2013 y STC 72/2013, ambas de 8 de abril, además de las STC $116 / 2013$ y STC 117/2013, de 20 de mayo. En todas ellas, al igual que ocurre en la STC 61/2013, el Tribunal alude a la doctrina que en su día estableció la STC 253/2004, de 22 de diciembre de 2004, que resuelve la cuestión de inconstitucionalidad 2045/1998 (Rec.2045/98) en cuanto a la protección social de una trabajadora a tiempo parcial, y en la que se pronunció sobre la constitucionalidad del art. 12.4 ET, declarando inconstitucional y nulo el párrafo segundo del citado artículo, en cuanto establecía que, para determinar los periodos de cotización exigidos a los trabajadores a tiempo parcial, para causar derecho a las prestaciones de Seguridad Social, incluida la de protección por desempleo, "se computarán exclusivamente las horas trabajadas" (FJ 9), en vez de considerar, como en el caso de los trabajadores a tiempo completo, cada día trabajado como un día cotizado, considerando que se vulnera el art. $14 \mathrm{CE}$ desde la perspectiva del derecho a la no discriminación, al generar una discriminación indirecta por razón de sexo.

${ }^{7}$ Primer párrafo del art. 247 LGSS.

${ }^{8}$ Dado que el art. 269.2 LGSS, establece que en el supuesto de que se hayan realizado trabajos a tiempo parcial, para determinar los períodos de cotización se estará a lo que se determine en la normativa reglamentaria de desarrollo, y que actualmente se contempla en el RD 625/1985.

${ }^{9}$ Ya que la aplicación de la normativa española da lugar a una discriminación indirecta y colisiona con la Directiva 79/7/CEE, de 19 diciembre 1978, sobre igualdad de trato en materia de Seguridad Social, aludida en la Sentencia del Tribunal de Justicia de la Unión Europea de 22 noviembre de 2012 (Asunto C-385/11), más conocida como Asunto Elbal Moreno, dictada a propósito de la cuestión prejudicial planteada por el Juzgado de lo Social núm. 33 de Barcelona, analiza el caso de una trabajadora, limpiadora de una comunidad de propietarios durante 18 años, en jornada parcial de 4 horas a la semana, es decir el 10\% de la jornada legal en España, a la que se le denegó la pensión de jubilación por la Entidad Gestora, en aplicación de la normativa correspondiente. 
modificaciones vienen a desarrollar el Acuerdo para la mejora de las condiciones de acceso a la protección social de los TTP ${ }^{10}$, firmado el 31 de julio de 2013 por el Ministerio de Empleo y Seguridad Social y las organizaciones CCOO, UGT, CEOE y CEPYME, meses después de que se dictase la STC 61/2013.

En base al mismo, la nueva regulación de la protección social de los TTP, así como a los fijos discontinuos, debe respetar los siguientes principios (MEYSS, 2016: 458):

- El "Principio de igualdad" en el acceso a las prestaciones, que se garantiza mediante la adaptación de las condiciones de acceso a las prestaciones para cada uno de los trabajadores en función de la mayor o menor parcialidad en el desarrollo de su actividad laboral, en línea con lo establecido respecto a su protección tanto en el Convenio sobre el trabajo a tiempo parcial, 1994 (núm. 175) ${ }^{11}$, como en la Directiva $97 / 81 / C E$, de 15 de diciembre de $1997^{12}$, que prestan una atención prioritaria a esta forma de trabajo.

- El "Principio de contributividad y proporcionalidad "entre las aportaciones realizadas mediante la cotización, "actividad realizada en cumplimiento de la ley en virtud de la cual sujetos obligados aportan recursos económicos a la Seguridad Social" (Blasco et al., 1996: 165), esto es, "la aportación dineraria que los sujetos obligados deben realizar, para el sostenimiento económico del Sistema" (Gorelli et al., 2015: 131), y las prestaciones a percibir, de manera que es de general aceptación que los trabajadores a jornada completa y a tiempo parcial causen prestaciones de cuantía adecuada en cada caso al esfuerzo contributivo realizado; lo que implica que debe existir una proporcionalidad [principio que

${ }^{10}$ En el que se postula una nueva regulación que facilite el reconocimiento de derechos a este colectivo: Período de carencia de 15 años en alta, independientemente del porcentaje de jornada del trabajador para tener derecho a la pensión de jubilación; mantenimiento para las pensiones de jubilación e IP del coeficiente multiplicador del 1,5 por cada día cotizado a tiempo parcial o como fijo-discontinuo y garantía del acceso a los complementos a mínimos en sus pensiones en los mismos términos que a los trabajadores a tiempo completo.

${ }_{11}$ Aplicable junto a la R182 - Recomendación sobre el trabajo a tiempo parcial, 1994 (núm. 182).

12 Por la que se incorpora al Derecho de la UE el Acuerdo Marco sobre el trabajo a tiempo parcial, celebrado el 6 de junio de 1997 entre las organizaciones interprofesionales de carácter general, la UNICE, la CES, y el CEEP. 
"en caso de no estar aparentemente reconocido, lo está a máximo nivel en la mayoría de los ordenamientos jurídicos" (Barnes,1998:19)] entre la prestación reconocida y la contribución económica realizada por los trabajadores ${ }^{13}$, por lo que es preciso seguir reforzándolo como elemento básico para la preservación del equilibrio financiero del sistema (MTIN, 2008: 120).

- El "Principio de conservación de normas favorables", que conduce a la necesidad de abordar la reforma respetando aquellos mecanismos que, no habiendo sido declarados inconstitucionales y nulos, tienen una valoración positiva, y por el que "la norma a aplicar es la más favorable para el trabajador, con independencia de su rango, sin perjuicio de que la norma pretérita o postergada siga formando parte del ordenamiento como un precepto válido" (Alonso y Casas, 1987: 698).

- El "Principio de solidaridad y suficiencia" de las pensiones, con la garantía de pensiones mínimas en la misma extensión, términos y condiciones que los establecidos para el resto de los trabajadores, siguiendo las Recomendaciones del Pacto de Toledo ${ }^{14}$ para las futuras reformas del sistema español de Seguridad Social.

- Y, el "Principio de legalidad", que impone la aprobación urgente de un nuevo marco legislativo que de cobertura legal a la actuación de la Administración Pública y garantice, al mismo tiempo, un tratamiento homogéneo y uniforme de estas situaciones ante los pronunciamientos judiciales.

A resultas de todo lo anteriormente expuesto, los cambios introducidos por el RD-L 11/2013, en la Disposición Adicional séptima del RDL 1/1994, de 20 de junio, se concretaron en un conjunto de medidas específicas, actualmente contempladas en los artículos 245 a 248 LGSS, modificando, por un lado, el artículo

${ }^{13}$ Así lo dictamina el Tribunal Constitucional en la Sentencia 156/2014, de 25 de septiembre de 2014, (FJ 5).

${ }^{14}$ Concretamente, la Recomendación número12, del Texto aprobado por la Comisión de Presupuestos sobre la base del informe elaborado por la Ponencia para el análisis de los problemas estructurales del sistema de la Seguridad Social y de las principales reformas que deberán acometerse, aprobado por el Pleno del Congreso de los Diputados en su sesión de 6 abril de 1995 (BOCG 12 de abril de1995). 
247 en toda su extensión, sobre el tratamiento de los períodos de cotización para acceder a las correspondientes prestaciones económicas, respecto de los periodos acreditados con contrato de trabajo a tiempo parcial, incluidos los contratos de trabajo fijodiscontinuo a tiempo parcial o completo, con independencia de que la reducción de jornada se realice en cómputo diario, semanal, mensual o anual, flexibilizando el número de años requeridos para acceder a una prestación, con la finalidad de garantizar en todo momento el principio de igualdad de los trabajadores, tanto para los de tiempo parcial como para los de tiempo completo ${ }^{15}$; y por otro, el artículo 248, en su apartado 3, donde se introdujo un cambio relevante respecto al porcentaje aplicable a la base reguladora de las prestaciones de jubilación e IP derivada de enfermedad común, sin alterar el resto.

\section{CÓMPUTO DE PERÍODOS DE COTIZACIÓN}

En lo que se refiere al tratamiento de los períodos de cotización, para calcular los períodos de carencia, se establece una nueva manera de computar el tiempo cotizado y así, acreditar los períodos de cotización necesarios a fin de causar derecho a las prestaciones de jubilación, IP, muerte y supervivencia, IT, maternidad y paternidad, con la voluntad de personalizar al máximo el nivel de parcialidad del trabajador a lo largo de toda su vida laboral y el propósito de concretar con una mayor precisión la totalidad de días que el trabajador ha prestado sus servicios a tiempo parcial, de manera que puedan ser computados para poder acceder a las diferentes prestaciones (Barcelón, 2013: 92 y ss.).

Para ello, en el artículo 247 LGSS, referente al cómputo de los periodos de cotización, se introducen dos términos novedosos, el "coeficiente de parcialidad" (en adelante, CP), como información esencial de la que se parte para el cálculo de los períodos de cotización en los TTP, y que viene determinado por la empresa al formalizar el alta del trabajador, quedando constancia en la vida laboral del mismo, y el "coeficiente global de parcialidad" (en adelante, CGP).

${ }^{15}$ Según Preámbulo del RD-L 11/2013, de 2 de agosto, para la protección de los trabajadores a tiempo parcial y otras medidas urgentes en el orden económico y social, en su Capítulo II. 


\section{Coeficiente de parcialidad}

De la actual regulación sobre las normas aplicables a los TTP se desprende que, el CP determina el porcentaje de cada período trabajado a jornada parcial y el CGP el porcentaje que representa el número de días efectivamente cotizados sobre el total de días en alta del trabajador, tanto a jornada parcial como a jornada completa.

De forma que, siguiendo lo que marca el apartado a) del artículo 247 LGSS, para computar el tiempo cotizado y acreditar los períodos de cotización necesarios a fin de causar derecho a las prestaciones, en primer lugar, debe tenerse en cuenta todos los periodos en los que el trabajador haya estado de alta con contrato a tiempo parcial, independientemente de la duración de la jornada en cada uno de los periodos trabajados.

En segundo lugar, se calcula un $\mathrm{CP}$, que viene determinado por el porcentaje de la jornada realizada a tiempo parcial respecto de la jornada realizada por un trabajador a tiempo completo comparable. Este coeficiente se aplica sobre cada uno de los períodos de alta con contrato a tiempo parcial. De tal forma que el CP de cada período es igual al porcentaje de jornada trabajada en el mismo.

En tercer lugar, y una vez determinado el CP, es traducido a días, aplicándolo en cada período al total de días cotizables. El resultado es el número de días efectivamente cotizados en cada uno de los períodos de alta con contrato a tiempo parcial.

Por último, si el trabajador tiene días trabajados a jornada completa, al número de días que resulten tras la aplicación del CP se le suman, en su caso, los días cotizados a tiempo completo, siendo el resultado, el total de días de cotización acreditados computables para el acceso a las prestaciones ${ }^{16}$.

${ }^{16} \mathrm{Al}$ que habrá que sumar si cabe, los días considerados como cotizados como consecuencia de situaciones de excedencia por cuidado de hijos (arts. 46.3 ET y 237, apartados 1 y 2 LGSS), así como los que se asignan por parto (art. 235 LGSS: 112 días completos de cotización por cada parto de un solo hijo y 14 días más por cada hijo a partir del segundo; aunque esta atribución legal solo es válida para las pensiones de jubilación e IP). No se sumarán en cambio, los días de reducción de jornada por cuidado de menor (art. 37.6 ET y 237.3 LGSS) que, al cotizarse a tiempo completo figuran como tales y no a tiempo parcial, incluyéndose en el cómputo del tiempo cotizado al $100 \%$, como si no existiera parcialidad. 


\section{Ejemplo:}

(A)Trabajador que acredita los siguientes períodos a tiempo parcial:

- Últimos 5840 días

(16 años) al $45 \%$

$$
\begin{aligned}
& C P=45 \\
& C P=50
\end{aligned}
$$

- 365 días (1 año) al 50\%.

Días efectivamente cotizados:

- 5840 * 45\% = 2628 días

- 365 * $50 \%=183$ días

Total días efectivamente cotizados: $2628+183=2811$

(B) - Además de estos períodos a tiempo parcial, el trabajador acredita 1095 días (3 años) de alta a tiempo completo, anteriores a estos períodos.

$(A+B)$ Total días acreditados de cotización efectiva: $1095+$ $2628+183=3906$

Si se calcula sobre el mismo supuesto los días acreditados de cotización efectiva, aplicando las reglas del RD-L 15/1998 y Real Decreto (en adelante, RD) 144/1999, de 29 de enero, por el que se desarrolla, en materia de acción protectora de la Seguridad Social, el RD-L 15/1998, y dónde se contempla en su artículo 3.1, que el número de horas efectivamente trabajadas por el trabajador a tiempo parcial se debe dividir entre cinco como divisor fijo [equivalente diario al cómputo de 1.826 horas anuales de la jornada estándar del trabajo a tiempo completo], el resultado es prácticamente igual:

\section{Ejemplo:}

(A) - Períodos cotizados trabajo tiempo parcial:

- Últimos 5840 días

(16 años) al $45 \%$ 13.147 horas

- 365 días (1 año) al 50\%. 913 horas

Total días efectivamente cotizados: 14.060 / $5=2.812$

(B) - Períodos cotizados a tiempo completo: 1095 días

$(\mathrm{A}+\mathrm{B})$ Total días acreditados de cotización efectiva: $1.095+$ $2.812=3.907$ 


\section{Coeficiente global de parcialidad}

Una vez determinado el CP, hay que establecer el CGP, que representa el número de días trabajados y acreditados como cotizados del trabajador, a lo largo de toda su vida laboral.

Fijados los días acreditados de cotización, y siguiendo con el apartado b) del artículo 247 LGSS, el cálculo del CGP es necesario para conocer el período mínimo de cotización exigido a los TTP para acceder a determinadas prestaciones económicas.

Este periodo es el resultado de aplicar al regulado con carácter general, el CGP, calculado este como el porcentaje que representa el número de días efectivamente cotizados sobre el total de días en alta del trabajador. Es decir, el porcentaje que representa el número de días trabajados y acreditados como efectivamente cotizados, de acuerdo con la regla anterior, sobre el total de días en alta del trabajador a lo largo de toda su vida laboral ${ }^{17}$, que se corresponden con los de vigencia de los diferentes tipos de contratos a tiempo parcial que haya tenido el trabajador, sin que ocurra lo mismo en el caso de trabajadores fijos-discontinuos, sean estos irregulares o periódicos, ya que esos días en alta corresponden con tiempos de actividad, a no ser que los días de inactividad hayan sido cubiertos por convenios especiales o por situaciones de desempleo (Barcelón, 2013: 97).

\section{Ejemplo:}

Siguiendo con el supuesto anterior,

(C) - Total días de cotización efectiva: $1.095+2628+183$ $=3.906$

(D) - Días en alta:

- 5.840 días (16 años) al 45\%

- 365 días (1 año) al 50\%

- 1.095 días (3 años) a jornada completa

Total días alta: 7.300

$$
\left(C^{\star} 100 / D\right) \text { - CGP: } 3.906 * 100 / 7.300=53,51 \%
$$

${ }_{17}$ Sin olvidar la regla especial en el cálculo del CGP que se realizará exclusivamente sobre los últimos 5 años en casos de IT o sobre los últimos 7 años o, en su caso, sobre toda la vida laboral (maternidad y paternidad). 


\section{Períodos de cotización exigibles para causar derecho a la prestación}

Para establecer los períodos de cotización, exigibles para causar derecho a las diferentes prestaciones, ha de tenerse en cuenta lo establecido en el apartado c) del artículo 247 LGSS, por el que, en el caso que se requiera que parte o la totalidad del período mínimo de cotización exigido, esté comprendido en un plazo de tiempo establecido, la determinación de la carencia viene dada por el resultado de aplicar al periodo regulado con carácter general el CGP, ignorándose los decimales resultantes de dicha operación.

De esta manera, el CGP "variará dependiendo de la ubicación temporal del trabajo a tiempo parcial y de su intensidad en esos tiempos" (Barcelón, 2013: 98), por lo que es independiente del tipo de contrato que tenga el trabajador en la fecha del hecho causante para tener derecho a la prestación correspondiente, salvo que durante todo el período de carencia exigido con carácter general, sea trabajador a jornada completa.

\section{Ejemplo:}

Siguiendo con el supuesto anterior, en caso de jubilación ordinaria, y teniendo en cuenta que el trabajador habrá de tenerla edad y el número de años cotizados exigible para poder jubilarse según el artículo 205.a) y la Disposición Transitoria sépti$\mathrm{ma}^{18}$, así como la carencia genérica y específica contempladas en el artículo 205. b) LGSS:

- Carencia genérica (5.475 días) y específica (730 días dentro de los 15 años anteriores al hecho causante)

(A) - • Últimos 5.840 días
(16 años) al $45 \%$
$\mathrm{CP}=45$
- 365 días (1 año) al 50\%.
$\mathrm{CP}=50$

Días efectivamente cotizados:

- 5840 * $45 \%=2.628$ días

${ }^{18}$ Aunque el legislador posibilita el acceso a la jubilación antes de cumplir la edad ordinaria en determinados supuestos como: Jubilación anticipada por razón del grupo o actividad profesional, por tener la condición de mutualista, sin tener la condición de mutualista, a partir de los 60 años, de trabajadores con discapacidad, jubilación parcial, o derivada del cese no voluntario en el trabajo. 
- 365 * $50 \%=183$ días

Total días efectivamente cotizados: $2628+183=2.811$

(B) - Además de estos períodos a tiempo parcial, el trabajador acredita 1.095 días (3 años) de alta a tiempo completo, anteriores a estos períodos.

$(\mathrm{A}+\mathrm{B})$ Total días acreditados de cotización efectiva: $1095+$ $2628+183=3.906$

El resultado de su CGP $=53,51 \%$

Carencia genérica:

5475 * 53,51\% $=2.930$ días

Carencia específica:

730 * 53,51\% = 391 días

En este caso, sí tendría derecho a la pensión de jubilación, por acreditar 3.906 días cotizados, número superior al exigido en la carencia genérica (2.930), de los que 391, se han cotizado dentro de los últimos quince años anteriores a la fecha del hecho causante.

Si en cambio, se calcula sobre el mismo supuesto, el período mínimo de cotización exigido a los TTP, aplicando las reglas del RD-L 15/1998 y RD 144/1999, que en su artículo 3.2 dispone la aplicación de un coeficiente multiplicador del 1,5 al tiempo de cotización acreditado [o días teóricos de cotización] durante los períodos de alta, el resultado es el siguiente:

\section{Ejemplo:}

(A) - Períodos cotizados trabajo tiempo parcial:

- Últimos 5840 días

(16 años) al 45\%

13.147 horas

- 365 días (1 año) al 50\%.

913 horas

Total días efectivamente cotizados: 14.060 / $5=2.812$

(B) - Períodos cotizados a tiempo completo: 1095 días

(A+B) Total días acreditados de cotización efectiva: 1.095+ $2.812=3.907$

(C) - Total días efectivamente cotizados: 14060 / $5=2812$ 
Aplicando el coeficiente de 1,5 2812 *1,5=4218

(D) - Días cotizados a tiempo completo: 1095 días

$(\mathrm{C}+\mathrm{D})$ Total días acreditados de cotización efectiva: $1095+$ $4218=5313$

Al ser $5313<5475$, el trabajador no tendría derecho a la prestación contributiva de la pensión de jubilación.

En este punto, hay que tener en cuenta que, para la determinación posterior de la cuantía de las pensiones de jubilación e IP derivada de enfermedad común, el número de días cotizados se incrementa con la aplicación del coeficiente del 1,5, sobre el número de días que se consideran efectivamente cotizados a tiempo parcial, sin que en ningún caso, el resultado supere los días en alta a tiempo parcial, como se estipula en el apartado 3 del artículo 248 LGSS.

\section{Ejemplo:}

Siguiendo con el supuesto anterior,

(A) -• Últimos 5.840 días

(16 años) al 45\%

$\mathrm{CP}=45$

- 365 días (1 año) al 50\%.

$\mathrm{CP}=50$

Días efectivamente cotizados:

- 5840 * $45 \%=2.628$ días

- 365 * $50 \%=183$ días

Total días efectivamente cotizados: $2628+183=2.811$

(B) - Además de estos períodos a tiempo parcial, el trabajador acredita 1.095 días (3 años) de alta a tiempo completo, anteriores a estos períodos.

(C) - Días efectivamente cotizados a tiempo parcial: 2811

Aplicando el coeficiente de 1,5 $2811 * 1,5=4217$

Al ser $4217<$ a los días en alta a tiempo parcial (5840), no supera el límite establecido legalmente.

(D) - Días cotizados a tiempo completo: 1095

$(\mathrm{C}+\mathrm{D})$ Total días cotizados: $4217+1095=5312$ 


\section{DETERMINACIÓN DE LA CUANTÍA DE LA PRESTACIÓN CONTRIBUTIVA}

Una vez reconocido el derecho a la prestación siguiendo las normas del artículo 247 LGSS, el siguiente paso es establecer su cuantía, que en su modalidad contributiva, se determina aplicando a la base reguladora el porcentaje correspondiente, relacionado con el período de cotización acreditado a lo largo de la vida laboral.

Para cuantificar la base reguladora, el RD-L 11/2013, no modifica la fórmula para su determinación, dado que los cambios que se efectúan sólo afectan al porcentaje aplicable a la misma a efectos de la determinación de la cuantía de la pensión de jubilación e IP derivada de enfermedad común.

En estos casos, la norma separa dos supuestos diferentes: que se acrediten 15 años de cotización efectiva o que se acredite un número de años inferior, ya que según el apartado 3 del artículo 248 LGSS, el porcentaje a aplicar sobre la base reguladora [variable en función de los años de cotización a la Seguridad Social], se realizará conforme a la escala general recogida en el artículo 210 LGSS $^{19}$, salvo si el interesado acredita un período de cotización inferior a quince años [considerando la suma de los días a tiempo completo con los días a tiempo parcial incrementados ya estos últimos con el coeficiente del 1,5], siendo entonces el mismo, el equivalente al que resulte de aplicar a 50 el porcentaje que represente el período de cotización acreditado por el trabajador sobre quince años.

${ }^{19}$ Y que ha sido modificado según la Recomendación $11^{\text {a }}$ del Pacto de Toledo, efectuada en la revisión del 2015 , con el propósito de responder a un esquema homogéneo de equiparación de los años cotizados con un mismo porcentaje. 


\section{Ejemplo:}

Siguiendo con el supuesto anterior,

(A) - Últimos 5.840 días

(16 años) al $45 \%$

$\mathrm{CP}=45$

- 365 días (1 año) al 50\%.

$\mathrm{CP}=50$

Días efectivamente cotizados:

- 5.840 * $45 \%=2.628$ días

- $365 * 50 \%=183$ días

Total días efectivamente cotizados: $2.628+183=2.811$

(B) - Además de estos períodos a tiempo parcial, el trabajador acredita 1.095 días (3 años) de alta a tiempo completo, anteriores a estos períodos.

(C) - Días efectivamente cotizados a tiempo parcial: 2811

Aplicando el coeficiente de 1,5 $2.811 * 1,5=4217$

(D) - Días cotizados a tiempo completo: 1.095

$(\mathrm{C}+\mathrm{D})$ Total días cotizados: $4.217+1.095=5.312$

Al ser $5.312<5.475$, el porcentaje a aplicar a la base reguladora será el resultado de aplicar el 50\% del porcentaje que representen 5.312 sobre los 5.475 días exigidos: 5.312 / 5.475 * $50=48,5 \%$ por lo tanto el $\%$ a aplicar sobre la respectiva base reguladora será del $48,5 \%$

Llegados a este punto, el hecho paradójico es que al trabajador a tiempo parcial, que tras la reforma [por la nueva manera de realizar los cálculos, aplicando el CP y el CGP] sí que cumple con la carencia exigida para tener derecho a la prestación contributiva por jubilación, se le aplica una penalización al no cubrir los 5.475 días.

Si el trabajador, en cambio, acreditase 5.475 días de cotización correspondientes a 15 años, el porcentaje a aplicar sobre la base reguladora, sería el establecido con carácter general. 


\section{Ejemplo:}

Siguiendo con el supuesto anterior,

(A) - • Últimos 6.205 días

$$
\text { (17 años) al 60\% CP }=60
$$

Días efectivamente cotizados:

- 6.205 * $60 \%=3.723$ días

Total días efectivamente cotizados: 3.723

(B) - Además de estos períodos a tiempo parcial, el trabajador acredita 1.095 días (3 años) de alta a tiempo completo, anteriores a estos períodos.

(C) - Días efectivamente cotizados a tiempo parcial: 3.102

Aplicando el coeficiente de 1,5

$3.723 * 1,5=5.585$

(D) - Días cotizados a tiempo completo: 1.095

(C+D) Total días cotizados: $5.585+1095=6.680$

Al ser $6.680>5475$, podría calcularse el porcentaje sobre la base reguladora conforme a lo establecido en la DT vigésima primera LGSS.

Por los 15 años

Por los 40 meses restantes

Porcentaje a aplicar sobre la respectiva base reguladora: $58,4 \%$

De esta manera, 2 trabajadores de igual antigüedad en una misma empresa pertenecientes a grupos profesionales distintos, que han cotizado por el mismo importe, con la diferencia que uno de ellos ha trabajado a tiempo parcial por el $60 \%$ de la jornada, y el otro al $45 \%$-exceptuando el año que trabajó al $50 \%$-, la diferencia de la pensión, habiendo contribuido ambos de la misma manera al sistema de la Seguridad Social, es en este caso, de un $9,9 \%$.

Todo esto implica que, aunque el TTP cumpla con la exigencia de carencia por los días cotizados a jornada parcial propia de su trayectoria laboral, para tener derecho a la prestación por jubilación, éste resulte penalizado por la aplicación de un porcentaje reductor sobre su base reguladora, de no cubrir sus periodos de 
cotización, la carencia genérica de 15 años que marca la regla general de estas prestaciones.

\section{CONCLUSIONES}

Aunque las modificaciones legislativas adoptadas tras la STC $61 / 2013$, facilitan el acceso a las prestaciones contributivas y, sobre todo, a las pensiones por parte de los TTP que, conforme a la regulación anterior hubieran tenido que acceder, si es caso, a las no contributivas, es indudable que, la aplicación del apartado 3 del artículo 248 LGSS, supone en muchos casos una disminución de su pensión, por lo que se sigue dejando en peor situación el derecho a la acción protectora de estos trabajadores.

En mi opinión, lejos de cumplirse las expectativas del Pacto de Toledo y del Acuerdo para la mejora de las condiciones de acceso a la protección social de los TTP, atendiendo más a la cantidad realmente ingresada al sistema de la Seguridad Social, desde un parámetro cuantitativo, que al periodo de cotización, desde un parámetro temporal, el esfuerzo de cotización sigue sin verse recompensado de igual manera en los TTP que en los trabajadores a tiempo completo.

\section{REFERENCIAS BIBLIOGRÁFICAS}

Alonso Olea, M. y Casas Bahamonde. M. (1987). Derecho del Trabajo. Madrid: Facultad de Derecho de la Universidad Complutense.

Barcelón Cobedo, S. (2013). Trabajo a tiempo parcial y Seguridad Social (Con las reformas introducidas por el RDL 11/2013). Valencia: Tirant lo Blanch.

Barnes, J. (1998). El principio de proporcionalidad. Estudio Preliminar. En Cuaderno de Derecho Público, 5. Madrid.

Blasco Lahoz, J.F., López Gandía, J., Momparler Carrasco, M.A. (1996).Curso de Seguridad Social. Valencia: Tirant lo Blanch.

Gorelli Hernández, J., Vílchez Porras, M., Álvarez Alcolea, M., De Val Tena, A. L., Gutiérrez Pérez, M. (2015). Lecciones de Seguridad Social. Madrid:Tecnos. 
Ministerio de Empleo y Seguridad Social. Secretaría de Estado de la Seguridad Social (2016). Informe sobre el desarrollo del Pacto de Toledo (2011-2015). Madrid.

Ministerio de Trabajo e Inmigración. Secretaría de Estado de la Seguridad Social (2008). Informe sobre el desarrollo del Pacto de Toledo (2003-2008). Madrid.

Nogueira Guastavino, M. (2012). El principio de igualdad y no discriminación en las relaciones laborales: Perspectiva constitucional reciente. En Lan Harremanak, 25.

\section{SENTENCIAS}

STC 156/2014, de 25 de septiembre de 2014.

STC 61/2013, de 14 de marzo de 2013.

STC 71/2013, de 8 de abril de 2013.

STC 72/2013, de 8 de abril de 2013.

STC 116/2013, de 20 de mayo de 2013.

STC 117/2013, de 20 de mayo de 2013.

STJUE de 22 de noviembre de 2012 (Asunto C-385/11).

STC 117/2011, de 4 de julio de 2011.

STC 79/2011, de 6 de junio de 2011.

STC 63/2011, de 16 de mayo de 2011.

STC 253/2004, de 22 de diciembre de 2004.

C.I. núm. 5862-2003, de 11 de noviembre de 2003.

\section{NORMATIVA:}

Real Decreto Legislativo 8/2015, de 30 de octubre, por el que se aprueba el texto refundido de la Ley General de la Seguridad Social.

Real Decreto Legislativo 2/2015, de 23 de octubre, por el que se aprueba el texto refundido de la Ley del Estatuto de los Trabajadores.

Ley $1 / 2014$, de 28 de febrero, para la protección de los trabajadores a tiempo parcial y otras medidas urgentes en el orden económico y social. 
Real Decreto-ley 11/2013, de 2 de agosto, para la protección de los trabajadores a tiempo parcial y otras medidas urgentes en el orden económico y social.

Directiva 2002/73/CE, de 23 de septiembre de 2002.

Real Decreto 144/1999, de 29 de enero, por el que se desarroIla, en materia de acción protectora de la Seguridad Social, el Real Decreto-ley 15/1998, de 27 de noviembre, de medidas urgentes para la mejora del mercado de trabajo en relación con el trabajo a tiempo parcial y el fomento de su estabilidad.

Real Decreto-ley 15/1998, de 27 de noviembre, de Medidas Urgentes para la Mejora del Mercado de Trabajo en Relación con el Trabajo a Tiempo Parcial y el Fomento de su Estabilidad.

Directiva 97/81/CE, de 15 de diciembre de 1997.

Directiva 97/80/CE, de 15 de diciembre de 1997.

Texto aprobado por el Pleno del Congreso de los Diputados y por la Comisión de Presupuestos en relación con el Informe de la ponenciapara el análisis de los problemas estructurales del sistema de la Seguridad Social y de las principales reformas que deberán acometerse, de 12 de abril de 1995.

Real Decreto Legislativo 1/1994, de 20 de junio, por el que se aprueba el Texto Refundido de la Ley General de la Seguridad Social.

Convenio sobre el trabajo a tiempo parcial, 1994 (núm. 175).

Recomendación sobre el trabajo a tiempo parcial, 1994 (núm. 182).

Real Decreto 625/1985, de 2 de abril, por el que se desarrolla la Ley 31/1984, de 2 de agosto, de Protección por Desempleo.

Constitución española, 1978.

Directiva 79/7/CEE, de 19 diciembre 1978. 